\title{
Pharyngeal Stenosis, CTCAE
}

National Cancer Institute

\section{Source}

National Cancer Institute. Pharyngeal Stenosis, CT CAE. NCI Thesaurus. Code C143757.

A disorder characterized by a narrowing of the pharyngeal airway. 